\title{
Matrix Element Calculations on the GPU
}

Joshua Isaacson, Enrico Bothmann, Walter Giele, Stefan Höche, Max Knobbe Based on arxiv:2106.06507

ML4Jets 2021 


\section{Motivation}

- LHC requires large number of Monte Carlo events

- Due to CPU costs, MC statistics will become significant uncertainty

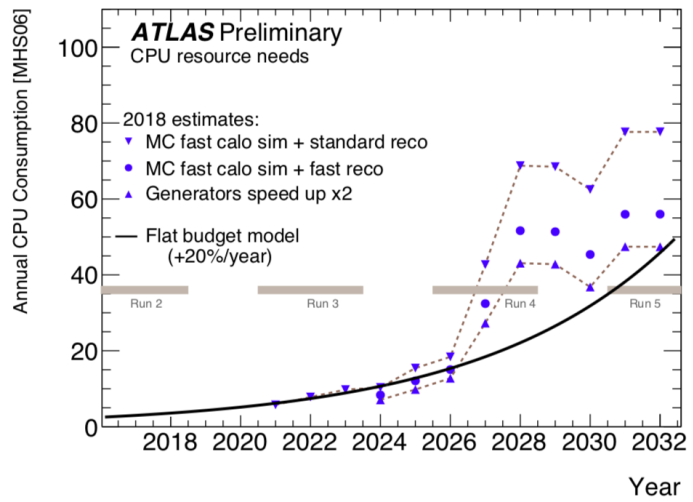

[ATLAS] 


\section{Motivation}
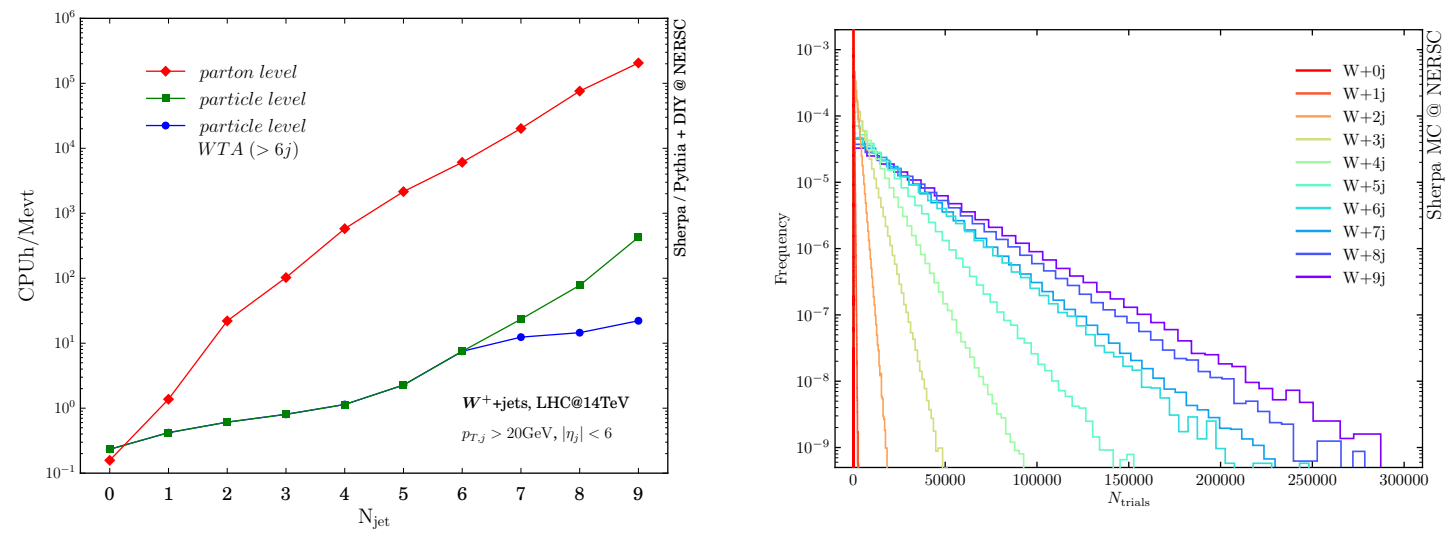

[S. Höche, S. Prestel, H. Schulz, 1905.05120]

- Time to generate an event dominated by hard process not shower

- Large computational cost for unweighting at high multiplicity 


\section{Works to Reduce Cost}

\section{Phase Space Generation from Samples}

- Requires a large sample before training

- GAN and VAE based [1707.0028, 1901.00875,

1901.05282, 1903.02433, 1907.03764, 1909.01359, 1909.04451,

$1912.08824,2008.06545,2008.08558$, etc.]

\section{Phase Space Generation from Random} Numbers

- Generates events as needed

- Normalizing flow based [2001.05478, 2001.05486, 2001.10028, 2104.04543]

- See talk from Timo Janßen from yesterday for more details 


\section{Phase Space Generation from Samples}

- Requires a large sample before training

- GAN and VAE based [1707.0028, 1901.00875,

1901.05282, 1903.02433, 1907.03764, 1909.01359, 1909.04451,

$1912.08824,2008.06545,2008.08558$, etc.]

\section{Phase Space Generation from Random} Numbers

- Generates events as needed

- Normalizing flow based [2001.05478, 2001.05486, 2001.10028, 2104.04543]

- See talk from Timo Janßen from yesterday for more details

Both approaches will benefit from improved event generation time 


\section{Recursive Matrix Element Generation}

Brends-Giele Recursion

- Reuse parts of calculation

- Most efficient for high multiplicity

- Reduces amplitude computations from $\mathcal{O}(n !)$ to $\mathcal{O}\left(3^{n}\right)$ for color-dressed and $\mathcal{O}\left(n^{3}\right)$ for color-ordered.

- $A(1, \ldots, n)=$ $J_{\mu}(n) p_{1, n}^{2} J^{\mu}(1, \ldots, n-1)$

[Nucl. Phys. B306(1988), 759] 


\section{Recursive Matrix Element Generation}

Brends-Giele Recursion

- Reuse parts of calculation

- Most efficient for high multiplicity

- Reduces amplitude computations from $\mathcal{O}(n !)$ to $\mathcal{O}\left(3^{n}\right)$ for color-dressed and $\mathcal{O}\left(n^{3}\right)$ for color-ordered.

- $A(1, \ldots, n)=$

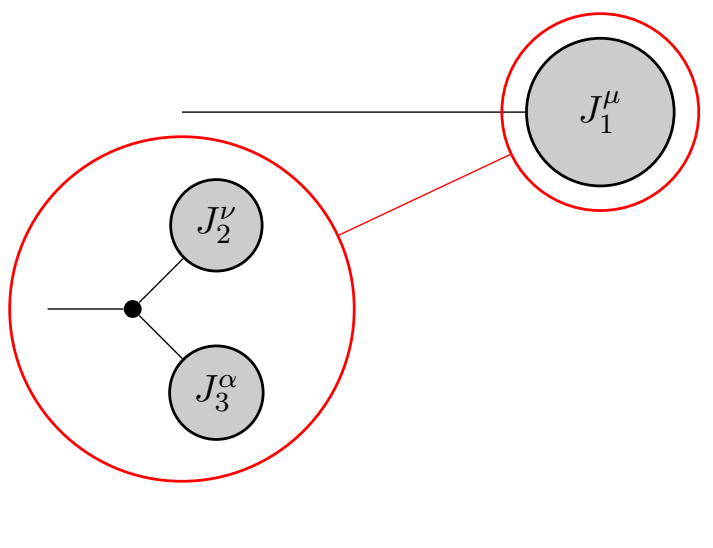
$J_{\mu}(n) p_{1, n}^{2} J^{\mu}(1, \ldots, n-1)$

[Nucl. Phys. B306(1988), 759] 


\section{Recursive Matrix Element Generation}

Brends-Giele Recursion

- Reuse parts of calculation

- Most efficient for high multiplicity

- Reduces amplitude computations from $\mathcal{O}(n !)$ to $\mathcal{O}\left(3^{n}\right)$ for color-dressed and $\mathcal{O}\left(n^{3}\right)$ for color-ordered.

- $A(1, \ldots, n)=$ $J_{\mu}(n) p_{1, n}^{2} J^{\mu}(1, \ldots, n-1)$

[Nucl. Phys. B306(1988), 759]

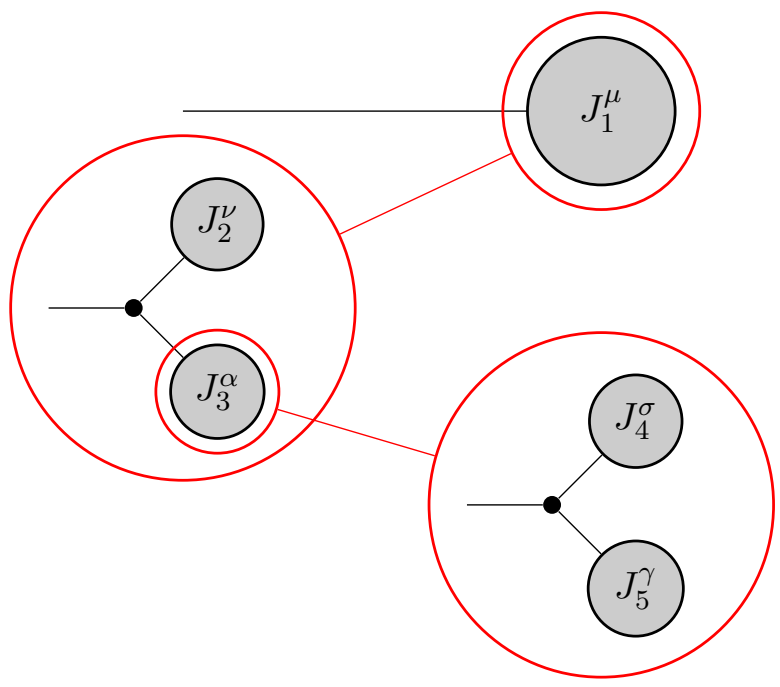




\section{Recursive Matrix Element Generation}

Brends-Giele Recursion

- Reuse parts of calculation

- Most efficient for high multiplicity

- Reduces amplitude computations from $\mathcal{O}(n !)$ to $\mathcal{O}\left(3^{n}\right)$ for color-dressed and $\mathcal{O}\left(n^{3}\right)$ for color-ordered.

- $A(1, \ldots, n)=$ $J_{\mu}(n) p_{1, n}^{2} J^{\mu}(1, \ldots, n-1)$

[Nucl. Phys. B306(1988), 759]

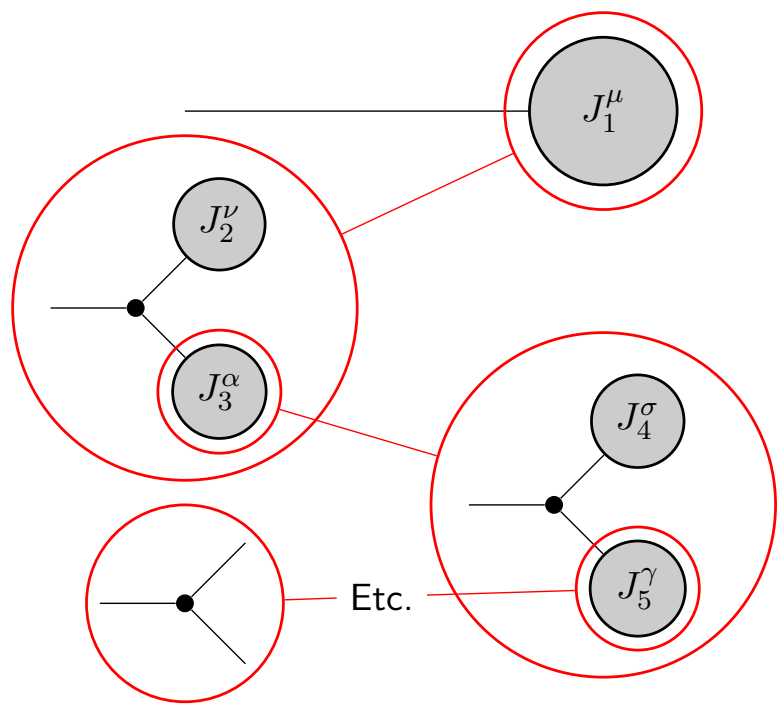




\section{Color-Ordered vs. Color-Dressed}

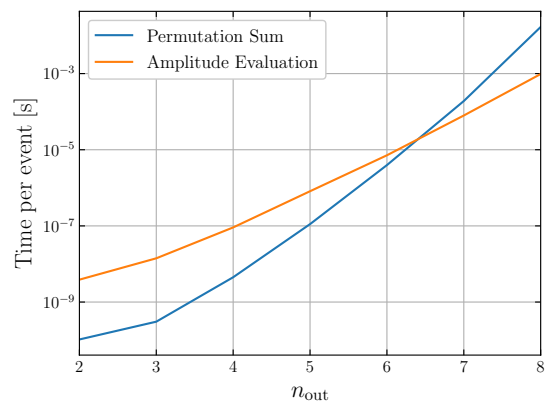

Scaling for Color-Ordered calculations

\section{Color-Ordered (CO):}

- Requires $(n-2) !^{2}$ color coefficients given by: $C_{\vec{\sigma} \vec{\sigma}^{\prime}}=$ $\sum_{a_{1} \ldots a_{n}}\left(F^{a_{\sigma_{2}}} \ldots F^{a_{\sigma_{n-1}}}\right)_{a_{1} a_{n}}\left(F^{a_{\sigma_{2}^{\prime}}} \ldots F^{a_{\sigma_{n-1}^{\prime}}}\right)_{a_{1} a_{n}}^{*}$

- Need to sum over all permutations to obtain full amplitude

\section{Color-Dressed (CD):}

- Color summed at each vertex. No need to sum over permutations

- Can sample color to Monte-Carlo the color sum

- Need to store color information of the gluons at each vertex 


\section{Why a GPU Implementation?}

Next-Gen Supercomputer Aurora:

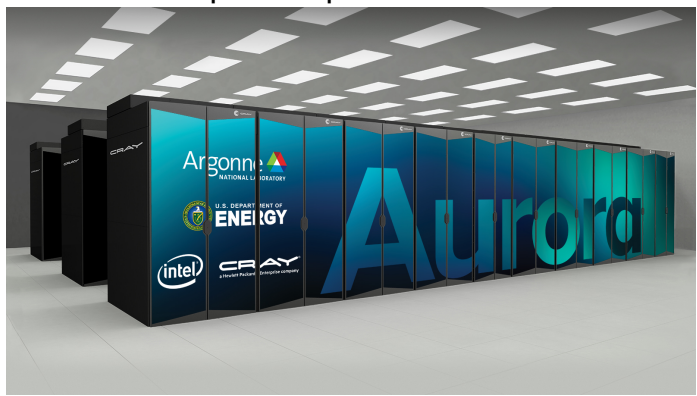

[https://alcf.anl.gov/aurora]
- Event generation is trivially parallelizable

- Aurora Compute Nodes:

- 2 Intel Xeon processors

- 6 Xeon arch-based GPUs

- Unified Memory

- Take advantage of modern supercomputer setups

- ML is already on GPUs, only missing piece is event generation 


\section{Memory Requirements}

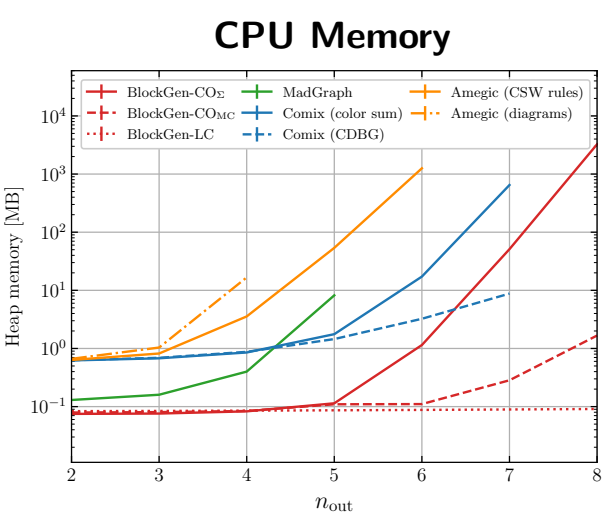

\section{GPU Memory}

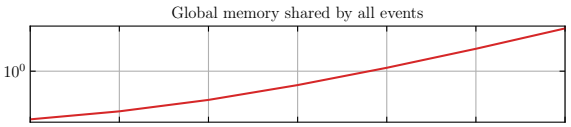

Global memory per event

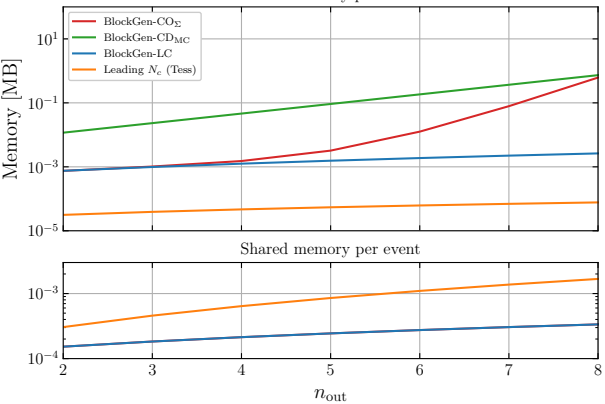




\section{Timings}

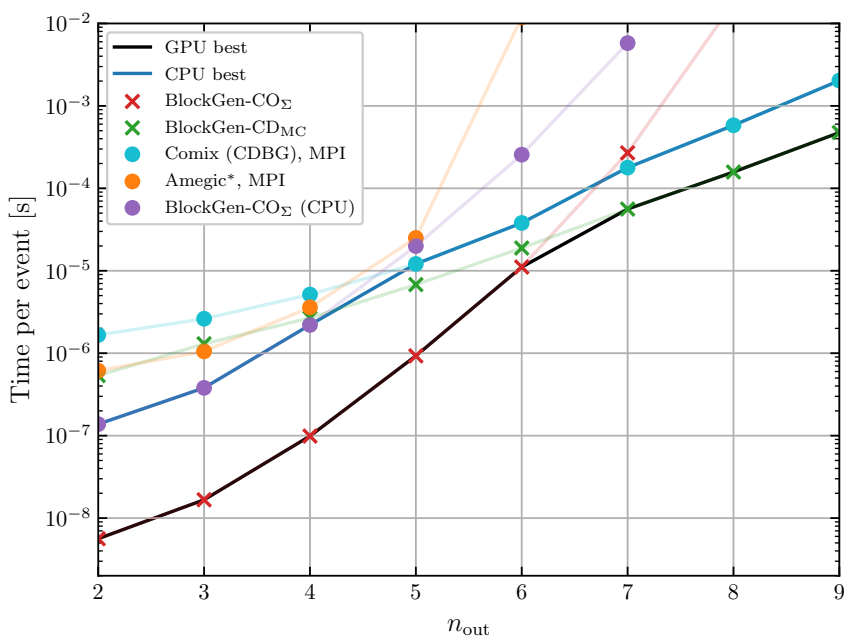

- CPU:

Intel ${ }^{\circledR} \mathrm{Xeon}^{\circledR}$

E5-2650 v2 8-core

(2.60 GHz, $20 \mathrm{MB}$ cache).

- GPU: NVIDIA V100

- CPUs are run with MPI with 16 threads to supply realistic chip-to-chip comparison 


\section{Future Steps}

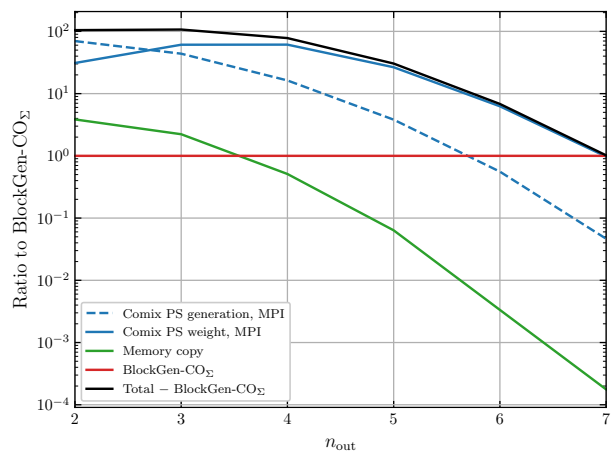

- Implement quarks and massive vector Bosons

- Develop hybrid calculation approach

- GPU Phase Space generator

- Generate PS with cuts on CPU

- Calculate PS weight and ME on GPU

- Need to ensure memory transfer is not the bottleneck 


\section{Conclusions}

\section{Cost of Event Generation:}

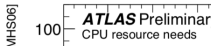

80-2018 estimates:

MC rast calo sim + standard reco

- MC fast calo sim + fast reco

$60-$ Generators speed up x2

- Flat budget model
$(+20 \% / y e a r)$

Fin2

$20-$

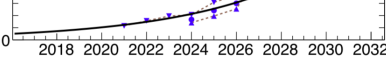

Year

- Matrix elements most expensive 


\section{Conclusions}

\section{Cost of Event Generation:}

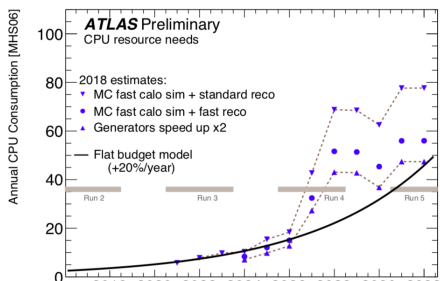

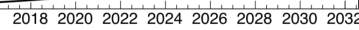

Year

- Matrix elements most expensive

\section{Brends-Giele:}

- Optimal generation

- Event generation trivially parallelizable

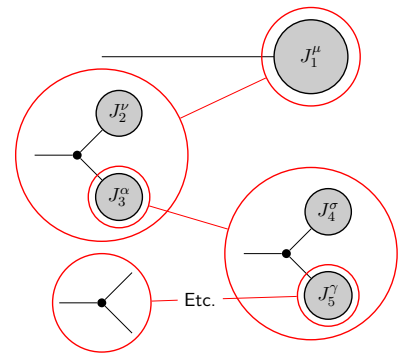




\section{Conclusions}

Cost of Event Generation:

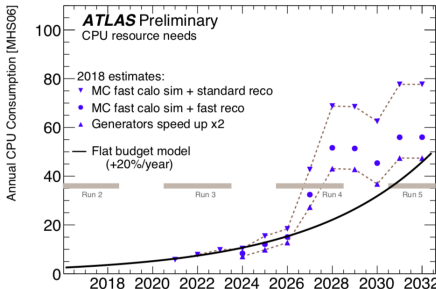

Year

- Matrix elements most expensive
Brends-Giele:

- Optimal generation

- Event generation trivially parallelizable

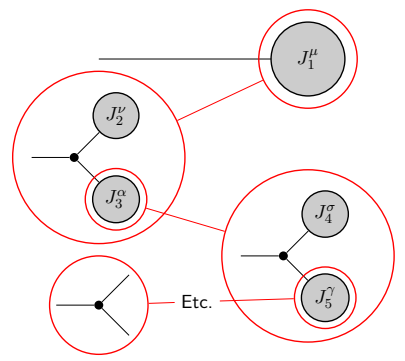

Results:

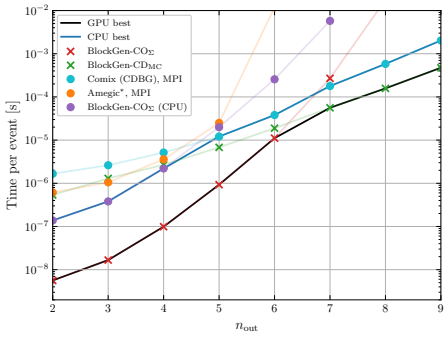

- Speedup of approximately a factor of 10

- BlockGen- $\mathrm{CO}_{\Sigma}$ is best for $n_{\text {out }}<7$ 